\title{
Characterization and Uniqueness of Distinguished Self-Adjoint Extensions of Dirac Operators
}

\author{
M. Klaus ${ }^{\star}$ and R. Wüst ${ }^{\star}$ \\ Departments of Physics and Mathematics, Princeton University, Princeton, New Jersey 08540, USA
}

\begin{abstract}
Distinguished self-adjoint extensions of Dirac operators are characterized by Nenciu and constructed by means of cut-off potentials by Wüst. In this paper it is shown that the existence and a more explicit characterization of Nenciu's self-adjoint extensions can be obtained as a consequence from results of the cut-off method, that these extensions are the same as the extensions constructed with cut-off potentials and that they are unique in some sense.
\end{abstract}

In the Hilbert space $H:=\left(L^{2}\left(\mathbb{R}^{3}\right)\right)^{4}$ the minimal Dirac operator of a spin $\frac{1}{2}$ particle with non-zero rest mass under the influence of a potential $q: \mathbb{R}_{+}^{3} \rightarrow \mathbb{R}\left(\mathbb{R}_{+}^{3}:=\mathbb{R}^{3} \backslash\{0\}\right)$ $q$ measurable, is given by

$$
\begin{aligned}
T & :=(\boldsymbol{\alpha} \cdot \mathbf{p}+\beta+q) \uparrow D_{0}, \\
D_{0} & :=\left(C_{0}^{\infty}\left(\mathbb{R}_{+}^{3}\right)\right)^{4}
\end{aligned}
$$

(cf. $[2,6,10]$ for more details).

We consider Coulomb like potentials $q$, i.e. potentials $q$ with $\mu:=\sup _{\mathbb{R}^{3}}|x q(x)|<\infty$.

Then $T$ is essentially self-adjoint if $\mu<\frac{1}{2} \sqrt{3}$ (cf. [6]) and in general not essentially self-adjoint if $\mu>\frac{1}{2} \sqrt{3}$.

But as long as $\mu<1$, physically distinguished self-adjoint extensions of $T$ still exist :

By means of cut-off potentials we have shown in [8-10], that for $q$ semibounded from above (or from below) and $\mu<1$

$$
\tilde{T}:=T^{*} \uparrow\left(D\left(T^{*}\right) \cap D\left(r^{-\frac{1}{2}}\right)\right)^{1}
$$

is a self-adjoint extension of $T$ (cf. the appendix for not semibounded potentials).

* On leave from Universität Zürich, Schöneberggasse 9, CH-8001 Zürich. Supported by Swiss National Science Foundation

$\star \star$ On leave from Technische Universität Berlin, Straße des 17. Juni 135, D-1000 Berlin

1 For $\alpha \in \mathbb{R}$ we denote by $r^{\alpha}$ the closure of the multiplication operator

$$
R_{\alpha}: D_{0} \rightarrow H, \quad u(x) \rightarrow|x|^{\alpha} u(x) \quad\left(u \in D_{0}, x \in \mathbb{R}_{+}^{3}\right) .
$$

The multiplication operators $q, r^{\alpha} q$ are defined analogously 
The physical interpretation of this operator $\tilde{T}$ is that all states in $D(\tilde{T})$ have finite potential energy. Obviously, $\tilde{T}$ is the unique self-adjoint extension of $T$ with this property (cf. the introduction in [4]). For if $S$ is a symmetric extension of $T$ with $D(S) \subset D\left(r^{-1 / 2}\right)$, then $S \subset \tilde{T}$ (cf. proof of the theorem).

If $q$ is not necessarily semibound, but still $\mu<1$ holds, Schmincke proved in [7] by another method that $\tilde{T}$ is self-adjoint (cf. Kalf [1] that $\tilde{T}$ is closed).

Finally, if

$$
\begin{aligned}
& T_{00}:=(\boldsymbol{\alpha} \cdot \mathbf{p}+\beta) \uparrow D_{0}, \\
& T_{0}:=\overline{T_{00}}
\end{aligned}
$$

denotes the operator of the free particle and $\mu<1$ is satisfied by $q$, Nenciu [4] showed that there exists a unique self-adjoint extension $\tilde{\tilde{T}}$ of $T$ such that

$$
D(\tilde{\tilde{T}}) \subset D\left(\left|T_{0}\right|^{1 / 2}\right)
$$

where the inclusion can be interpreted as the fact that only states with finite kinetic energy are in the domain of $\tilde{\tilde{T}}$.

We show in this paper that in the case of semibounded potentials $q$ with $\mu<1$ the existence and uniqueness of such a self-adjoint operator $\tilde{\tilde{T}}$ is an easy consequence of the results in [10] and that $\tilde{T}$ is explicitly given and equal to $\tilde{T}$.

The results are still valid without the assumption of semiboundness of $q$ as we sketch in the appendix. But, since the case where $\lim _{x \rightarrow 0} q(x)=-\varlimsup_{x \rightarrow 0} q(x)=\infty$ has no physical interest we restricted ourselves in $[9,10]$ and in the main part of this paper on semibounded potentials.

Theorem. Let $q: \mathbb{R}_{+}^{3} \rightarrow \mathbb{R}$ be a measurable function, semibounded from above or from below and

$$
\mu:=\sup _{\mathbb{R}_{+}^{3}}|x q(x)|<1 .
$$

\section{Denote}

$$
\begin{aligned}
& \tilde{T}:=T^{*} \uparrow\left(D\left(T^{*}\right) \cap D\left(r^{-1 / 2}\right)\right) \\
& \tilde{T}:=T^{*} \uparrow\left(D\left(T^{*}\right) \cap D\left(\left|T_{0}\right|^{1 / 2}\right)\right)
\end{aligned}
$$

( $T, T_{0}$ as above).

Then $\tilde{T}, \tilde{T}$ are self-adjoint and

$$
\tilde{T}=\tilde{T} \text {. }
$$

Moreover, $\tilde{T}$ and $\tilde{\tilde{T}}$ are the unique self-adjoint extensions of $T$ with domain contained in $D\left(r^{-1 / 2}\right)$ or $D\left(\left|T_{0}\right|^{1 / 2}\right)$, resp.

First, we prove the following

Proposition. Under the assumptions and with the notations of the theorem

$$
D(\tilde{T}) \subset D\left(\left|T_{0}\right|^{1 / 2}\right)
$$

holds. 
Proof. Let $\left\{q_{t}\right\}_{t>0}$ be a family of bounded cut-off potentials as in [10, Theorem 4] and

$$
T_{t}:=\overline{T_{00}+q_{t}} \quad(t>0) .
$$

Then $\left\{T_{t}\right\}_{t>0}$ has a strong resolvent limit which is self-adjoint and equal to $\tilde{T}[8$, $10]$.

The multiplication operators $r q_{t}(t>0)$ are uniformly bounded and

$$
s-\lim _{t \rightarrow \infty} \overline{r q}=\overline{r q} \text {. }
$$

$r^{-1 / 2}$ and $r^{-1}$ are relatively bounded with respect to $T_{0}$ and so with respect to $\left|T_{0}\right|$ (cf. [9, Lemma 3] with $q=0$, or [10, Lemma 3] and [2, $\S$ V.5.4]).

$r^{-1 / 2}$ is also relatively bounded with respect to $\tilde{T}$. For, if $u \in D(\tilde{T})$ then there exists a family $\left\{u_{t}\right\}_{t>0}$ with $\lim _{t \rightarrow \infty} u_{t}=u$ and $\lim _{t \rightarrow \infty} T_{t} u_{t}=\tilde{T} u$. But then $\left\{r^{-1 / 2} u_{t}\right\}_{t>0}$ is weakly convergent to $r^{-1 / 2} u$ (see the 2 nd step in the proof of the theorem in [9]), thus $\left\|r^{-1 / 2} u\right\| \leqq \underline{\lim }\left\|r^{-1 / 2} u_{t}\right\|$. By Lemma 3 in [9] we have

$$
(1-\mu)\left\|r^{-1 / 2} u_{t}\right\| \leqq\left\|T_{t} u_{t}\right\|+2\left\|u_{t}\right\| \quad(t>0)
$$

and so

$$
(1-\mu)\left\|r^{-1 / 2} u\right\| \leqq\|\tilde{T} u\|+2\|u\| \quad(u \in D(\tilde{T})) .
$$

Moreover, 0 is in the resolvent set of $T_{0}, T_{i}(t>0)$ and $\tilde{T}$ (cf. [10]). Therefore the following operators are everywhere defined and bounded:

$$
\begin{aligned}
& r^{-1} T_{0}^{-1}, \quad\left(r^{-1} T_{0}^{-1}\right)^{*}=\overline{T_{0}^{-1} r^{-1}}, \\
& r^{-1}\left|T_{0}\right|^{-1}, \\
& r^{-1 / 2}\left|T_{0}\right|^{-1 / 2}, \quad\left(r^{-1 / 2}\left|T_{0}\right|^{-1 / 2}\right)^{*}=\overline{\left|T_{0}\right|^{-1 / 2} r^{-1 / 2}}, \\
& \text { (cf. [5, Theorem X.18]) } \\
& r^{-1 / 2} T_{0}^{-1}, \quad\left(r^{-1 / 2} T_{0}^{-1}\right)^{*}=\overline{T_{0}^{-1} r^{-1 / 2}}, \\
& r^{-1 / 2} \tilde{T}^{-1} .
\end{aligned}
$$

Using (3), (5), the strong resolvent convergence of $\left\{T_{t}\right\}_{t>0}$ and the second resolvent equation we have for $u \in H$

$$
\begin{aligned}
& \left\|\left(\tilde{T}^{-1}-T_{0}^{-1}+\overline{T_{0}^{-1} r^{-1}} \overline{r q} \tilde{T}^{-1}\right) u\right\| \\
& =\lim _{t \rightarrow \infty}\left\|\left(-T_{0}^{-1} q_{t} T_{t}^{-1}+\overline{T_{0}^{-1} r^{-1}} \overline{r q} \tilde{T}^{-1}\right) u\right\|= \\
& \leqq \\
& \varlimsup_{t \rightarrow \infty}\left\|\overline{T_{0}^{-1} r^{-1}}\right\|\left(\left\|\overline{r q}_{t}\right\|\left\|T_{t}^{-1} u-\tilde{T}^{-1} u\right\|\right. \\
& \left.\quad+\left\|\left(\overline{r q}_{t}-\overline{r q}\right) \tilde{T}^{-1} u\right\|\right) \\
& =0,
\end{aligned}
$$

which gives the representation

$$
\tilde{T}^{-1}=T_{0}^{-1}-\overline{T_{0}^{-1} r^{-1}} \overline{r q} \tilde{T}^{-1} \text {. }
$$


For $u \in H$ and $v \in D_{0}$

$$
\begin{aligned}
& \left.\left.\overline{\left(T_{0}^{-1} r^{-1}\right.} \overline{r q} \tilde{T}^{-1} u, T_{00} v\right)-\overline{\left(T_{0}^{-1} r^{-1 / 2}\right.} \overline{r q} r^{-1 / 2} \tilde{T}^{-1} u, T_{00} v\right) \\
& \quad=\left(\overline{r q} \tilde{T}^{-1} u, r^{-1} v\right)-\left(r^{-1 / 2} \overline{r q} \tilde{T}^{-1} u, r^{-1 / 2} v\right) \\
& \quad=0 .
\end{aligned}
$$

Since $T_{00} D_{0}$ is dense in $H$,

$$
\overline{T_{0}^{-1} r^{-1}} \overline{r q} \tilde{T}^{-1}=\overline{T_{0}^{-1} r^{-1 / 2}} \overline{r q} r^{-1 / 2} \tilde{T}^{-1} .
$$

The Eqs. (6) and (7) together with the inclusion

$$
R\left(T_{0}^{-1}\right)=D\left(T_{0}\right)=D\left(\left|T_{0}\right|\right) \subset D\left(\left|T_{0}\right|^{1 / 2}\right)
$$

show that it is sufficient for (2) to prove

$$
R \overline{\left(T_{0}^{-1} r^{-1 / 2}\right)} \subset D\left(\left|T_{0}\right|^{1 / 2}\right) \text {. }
$$

But by the functional calculus for self-adjoint operators (cf. [5, VII])

$$
\left|T_{0}\right|^{1 / 2} T_{0}^{-1}\left|T_{0}\right|^{1 / 2}
$$

is a densely defined and bounded operator, therefore

$$
\begin{aligned}
T_{0}^{-1} & =\left|T_{0}\right|^{-1 / 2}\left|T_{0}\right|^{1 / 2} T_{0}^{-1}\left|T_{0}\right|^{1 / 2}\left|T_{0}\right|^{-1 / 2} \\
& =\left.\left|T_{0}\right|^{-1 / 2}\left|\overline{\left.T_{0}\right|^{1 / 2} T_{0}^{-1}\left|T_{0}\right|^{1 / 2}}\right| T_{0}\right|^{-1 / 2}
\end{aligned}
$$

and with (5)

$$
\overline{T_{0}^{-1} r^{-1 / 2}}=\left|T_{0}\right|^{-1 / 2}\left|\overline{\left.T_{0}\right|^{1 / 2} T_{0}^{-1}\left|T_{0}\right|^{1 / 2}}\right| \overline{\left.T_{0}\right|^{-1 / 2} r^{-1 / 2}},
$$

which proves (8).

Proof of the Theorem. $r^{-1 / 2}$ is relatively bounded with respect to $\left|T_{0}\right|^{1 / 2}$, therefore

$$
D\left(\left|T_{0}\right|^{1 / 2}\right) \subset D\left(r^{-1 / 2}\right) \text {. }
$$

Together with the proposition this inclusion gives

$$
\begin{aligned}
D(\tilde{\tilde{T}}) & =\left(D\left(T^{*}\right) \cap D\left(\left|T_{0}\right|^{1 / 2}\right)\right) \subset\left(D\left(T^{*}\right) \cap D\left(r^{-1 / 2}\right)\right) \\
& =D(\tilde{T})=\left(D\left(T^{*}\right) \cap D(\tilde{T})\right) \subset\left(D\left(T^{*}\right) \cap D\left(\left|T_{0}\right|^{1 / 2}\right)\right)=D(\tilde{T}),
\end{aligned}
$$

which proves (1).

The self-adjointness of $\tilde{T}$ is proved in [10]. Finally, since every symmetric extension of $T$ is a restriction of $T^{*}$ and therefore every symmetric extension $S$ of $T$ with $\underset{\approx}{D}(S) \subset D\left(r^{-1 / 2}\right)$ or $D(S) \subset D\left(\left|T_{0}\right|^{1 / 2}\right)$ has to be a restriction of $\tilde{T}$ or $\tilde{\tilde{T}}$ resp., $\tilde{T}$ and $\tilde{T}$ are the unique self-adjoint extensions with these properties.

\section{Appendix}

We sketch the way how the results can be proved in the case of a nonsemibounded potential $q$ by means of a double cut-off procedure. 
Let $q: \mathbb{R}_{+}^{3} \rightarrow \mathbb{R}$ be a measurable function and $\mu:=\sup _{\mathbb{R}_{+}^{3}}|x q(x)|<1$.

\section{Denote}

$$
\begin{aligned}
& q^{(\tau)}(x):=\min \{\tau, q(x)\} \quad\left(x \in \mathbb{R}_{+}^{3}, \tau>0\right) \\
& q_{t}^{(\tau)}(x):=\max \left\{-t, q^{(\tau)}(x)\right\} \quad\left(x \in \mathbb{R}_{+}^{3}, \tau>0, t>0\right) \\
& T_{t}^{(\tau)}:=T_{0}+q_{t}^{(\tau)} \quad(t, \tau>0) \\
& T^{(\tau)}:=T_{00}+q^{(\tau)} \quad(\tau>0) \\
& T::=\mathrm{T}_{00}+\mathrm{q} \text {. }
\end{aligned}
$$

For each $\tau>0$ we can apply Theorem 4 in [10], which shows that the strong graph limit $\tilde{T}^{(\tau)}$ of the family $\left\{T_{t}^{(\tau)}\right\}_{t>0}$ exists, is equal to the strong resolvent limit of $\left\{T_{t}^{(\tau)}\right\}_{t>0}[8]$, that

$$
\tilde{T}^{(\tau)}=T^{(\tau) * \uparrow\left(D\left(T^{(\tau) *}\right) \cap D\left(r^{-1 / 2}\right)\right)}
$$

and $\tilde{T}^{(\tau)}$ is a self-adjoint extension of $T^{(\tau)}$.

Moreover, we have

$$
\begin{aligned}
& D\left(\tilde{T}^{(\tau)}\right)=\text { constant } \quad(\tau>0), \\
& \overline{\tilde{T}^{(\tau)}-\tilde{T}^{\left(\tau^{\prime}\right)}}=\overline{q^{(\tau)}-q^{\left(\tau^{\prime}\right)}} \quad\left(\tau, \tau^{\prime}>0\right)
\end{aligned}
$$

and

$$
\left\|\tilde{T}^{(\tau)} u\right\| \geqq \frac{1}{1+\mu} \sqrt{1-\mu^{2}}\|u\| \quad\left(u \in D\left(\tilde{T}^{(\tau)}\right), \tau>0\right)
$$

(cf. [10, Theorem 5]).

This allows us to apply the convergence theorem [8], and [10, Theorem 1] a second time, now with respect to the family $\left\{\tilde{T}^{(\tau)}\right\}_{\tau>0}$. Thus, the strong graph limit

$$
\hat{T}:=g-\lim T^{(\tau)}
$$

exists, is a self-adjoint extension of $T$ and has at least the spectral gap $\left(-\frac{1}{1+\mu} \sqrt{1-\mu^{2}}, \frac{1}{1+\mu} \sqrt{1-\mu^{2}}\right)$ (see [3] for a best possible result). Since (4) holds for $\tilde{T}^{(\tau)}$ uniformly in $\tau>0$, the argument in the second step of the proof of the theorem in [9] shows

$$
D(\hat{T}) \subset D\left(r^{-1 / 2}\right) \text {. }
$$

By definition of $\hat{T}$, for every $u \in D(\hat{T})$

$$
\begin{aligned}
& u=\lim _{\tau \rightarrow \infty} \lim _{t \rightarrow \infty} \lim _{n \rightarrow \infty} u_{t, n}^{(\tau)}, \\
& \hat{T} u=\lim _{\tau \rightarrow \infty} \lim _{t \rightarrow \infty} \lim _{n \rightarrow \infty} T_{t}^{(\tau)} u_{t, n}^{(\tau)}
\end{aligned}
$$

hold with a suitable family $\left\{u_{t, n}^{(\tau)}\right\}_{\substack{\tau>0 \\ t>0 \\ n \in \mathbb{N}}}$ in $D_{0}$. 
Then the method in the first step of the proof of the theorem in [9] allows us to show that

$$
D\left(T^{*}\right) \cap D\left(r^{-1 / 2}\right) \subset D(\hat{T}) .
$$

From this and (9)

$$
\hat{T}=T^{*} \uparrow\left(D\left(T^{*}\right) \cap D\left(r^{-1 / 2}\right)\right)=: \tilde{T}
$$

follows.

To get the results of the theorem in this paper for a nonsemibounded potential $q$, we only need to show that (4) and (6) are still true for the above operator $\tilde{T}$. (4) can be proved in the same way, because it is true for $\tilde{T}^{(\tau)}$, uniformly in $\tau>0$. (6) follows immediately from the fact that the multiplication operators $r q_{t}^{(\tau)}(t>0, \tau>0)$ are uniformly bounded in $t, \tau>0$ that

$$
s-\lim _{\tau \rightarrow \infty} s-\lim _{t \rightarrow \infty} \overline{r q_{t}^{(\tau)}}=\overline{r q}
$$

and

$$
s-\lim _{\tau \rightarrow \infty} s-\lim _{t \rightarrow \infty} T_{t}^{(\tau)^{-1}}=s-\lim _{\tau \rightarrow \infty} \tilde{T}^{(\tau)^{-1}}=\tilde{T}^{-1} .
$$

Acknowledgements. The authors would like to thank B. Simon for stimulating discussions and Princeton University for her kind hospitality.

\section{References}

1. Kaif, H.: The Virial theorem in relativistic quantum mechanics. J. Funct. Anal. 21, 389-396 (1976)

2. Kato, T.: Perturbation theory for linear operators. Berlin, Heidelberg, New York: Springer 1966

3. Klaus, M., Wuist, R. : Spectral properties of Dirac operators with singular potentials. (to appear)

4. Nenciu, G. : Self-adjointness and invariance of the essential spectrum for Dirac operators defined as quadratic forms. Commun. math. Phys. 48, 235-247 (1976)

5. Reed, M., Simon, B.: Methods of modern mathematical physics, Vol. I and II. New York: Academic Press 1972 and 1975

6. Schmincke, U.-W. : Essential self-adjointness of Dirac operators with a strongly singular potentıal. Math. Z. 126, 71-81 (1972)

7. Schmincke, U.-W.: Distinguished self-adjoint extensions of Dirac operators. Math. Z. 129, 335-349 (1972)

8. Wüst, R. : A convergence theorem for self-adjoint operators applicable to Dirac operators with cutoff potentials. Math. Z. 131, 339-349 (1973)

9. Wüst, R.: Distinguished self-adjoint extensions of Dirac operators constructed by means of cut-off potentials. Math. Z. 141, 93-98 (1975)

10. Wist, R.: Dirac operators with strongly singular potentials. Math. Z. 152, 259-271 (1977)

Communicated by J. Ginibre

Received August 21, 1978 